\title{
Insights into the Enhanced Catalytic Activity of Cytochrome c When Encapsulated in a Metal-Organic Framework
}

Yijing Chen, Felipe Jiménez-Angeles, Baofu Qiao, Matthew D. Krzyaniak, Fanrui Sha, Satoshi

Kato, Xinyi Gong, Cassandra T. Buru, Zhijie Chen, Xuan Zhang, Nathan C. Gianneschi, Michael R. Wasielewski, Monica Olvera de la Cruz, ${ }^{*}$ Omar K. Farha*

* To whom correspondence should be addressed.

Email:*o-farha@northwestern.edu

*m-olvera@northwestern.edu

Table of Contents

$\begin{array}{lll}\text { A. } & \text { Materials and Methods } & \text { S2 } \\ \text { B. Figures } & \text { S3 } \\ \text { C. References } & \text { S8 }\end{array}$ 


\section{Supporting Information}

\section{A. Materials and Methods}

\section{Materials.}

Cytochrome c from bovine heart (CAS number: 9007-43-6, catalog number: C3131); 2,2'-Azinobis(3-ethylbenzothiazoline-6-sulfonic acid) diammonium salt (CAS number: 30931-67-0, part number: A9941); was produced from Sigma-Aldrich, USA. Tris-HCl buffer solution (1 M, $\mathrm{pH}=7.5,1 \mathrm{~L}$, catalog number: 15-567-027) was purchased from Fisher Scientific, USA. Hydrogen peroxide solution MOF NU-1000 was synthesized and activated following the published method. ${ }^{1}$ $20 \mathrm{mg}$ NU-1000 crystals were soaked in $7 \mathrm{mg} / \mathrm{ml}$ Cyt c solution for 5 hours to ensure its encapsulation and Cyt c@NU-1000 crystals were then centrifuged down and washed for 3 times with deionized (DI) water to remove the excess enzymes.

\section{Physical Methods and Measurements.}

Powder X-ray diffraction was measured at room temperature on a STOE-STADI P powder diffractometer equipped with an asymmetric curved Germanium monochromator (CuK $\alpha 1$ radiation, $\lambda=1.54056 \AA$ ) and a one-dimensional silicon strip detector (MYTHEN2 1K from DECTRIS). The line focused $\mathrm{Cu}$ X-ray tube was operated at $40 \mathrm{kV}$ and $40 \mathrm{~mA}$. Ar sorption isotherm measurements were performed on a Micromeritics ASAP-2020 (Micromeritics, Norcross, GA) at $87 \mathrm{~K}$. The data points between 0.03 and $0.14 \mathrm{P} / \mathrm{P} 0$ were chosen for the BET surface area calculation to minimize the error for consistency criteria ( $\mathrm{R} 2=0.9997)$. Between 30 and $50 \mathrm{mg}$ of material was used for each measurement. The pore size distribution analysis was obtained by fitting the 2D-NLDFT model to the argon adsorption isotherms measured at $87 \mathrm{~K}$. Inductively coupled plasma-optical emission spectroscopy (ICP-OES) was performed three times to measure the quantify the loading of Cyt $\mathrm{c}$ in NU-1000. The experiment was performed on QTEGRA software v. 2.2 Thermo iCap 7600 Duo ICP-OES (Thermo Fisher Scientific, Waltham, MA, USA) operating in standard mode. $\mathrm{HNO}_{3}(1.5 \mathrm{~mL})$ was added to 2-3 mg Cyt $\mathrm{c} @ \mathrm{NU}-1000$ in a $5 \mathrm{~mL}$ microwave vial for digestion followed by $0.5 \mathrm{~mL} \mathrm{H}_{2} \mathrm{O}_{2}$. After sealing, the vial was heating in a Biotage (Uppsala, Sweden) SPX microwave reactor (software version 2.3, build 6250) at 150 ${ }^{\circ} \mathrm{C}$ for $15 \mathrm{~min}$. The solution was then diluted by Millipore water, and the ratio of $\mathrm{Zr}$ to $\mathrm{S}$ was determined by calibration curve of standard solutions. Diffuse reflectance UV-vis spectra of the 


\section{Supporting Information}

solid MOF samples were recorded with a Shimadzu UV-3600 with a Harrick Praying Mantis diffuse reflectance accessory. Teflon was used as a perfect reflector for baseline collection and the samples were diluted with Teflon for the measurements. The obtained reflectance spectra were converted to absorption spectra by using Kubelka-Munk function. $\alpha / S=(1-R)^{2}(2 R)^{-1}$ where $R$ is the reflectance, $\alpha$ and $\mathrm{S}$ are the absorption and scattering coefficients, respectively. ITC experiments were performed using a VP-ITC titration microcalorimeter (MicroCal Inc.). ${ }^{2}$ STEMEDX analysis was performed using EPIC TEM JEOL ARM200CF operated at $200 \mathrm{keV}$ and were acquired with probe size $8 \mathrm{C}$ and camera length $20 \mathrm{~cm}$.

\section{B. Figures}

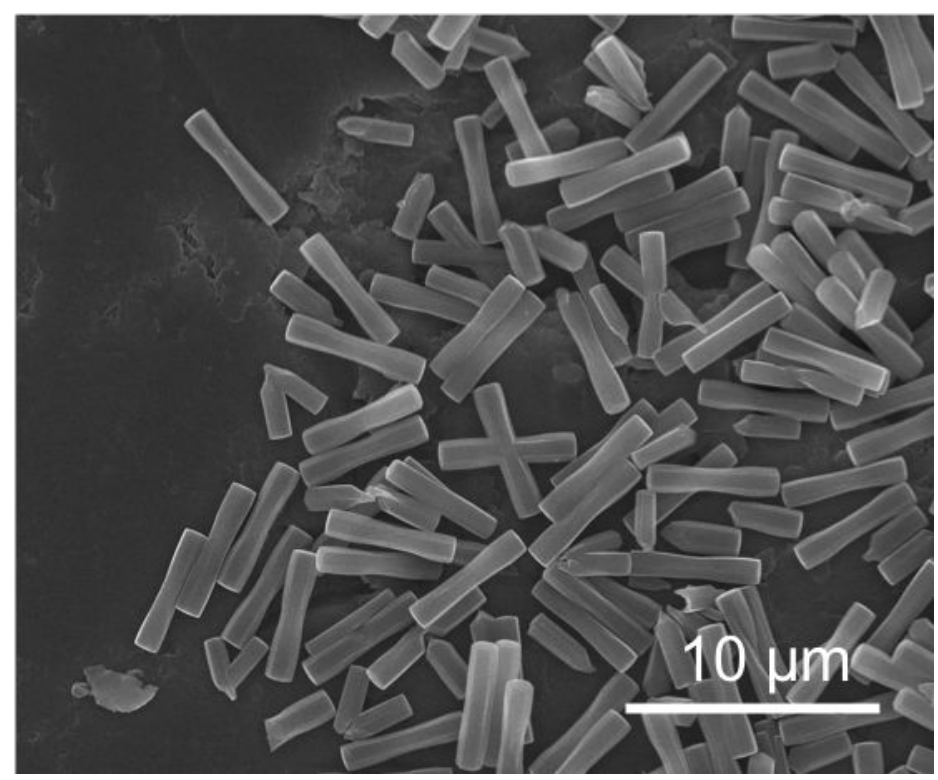

Figure S1. SEM image of NU-1000. 


\section{Supporting Information}

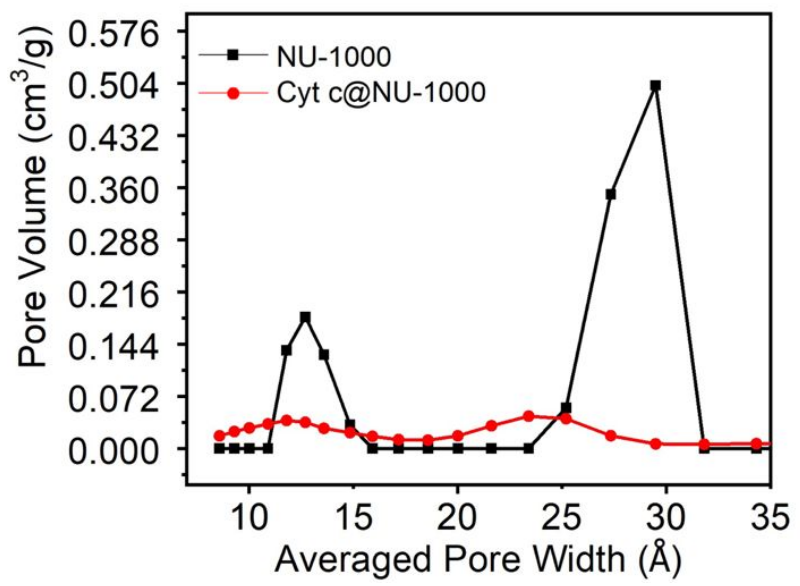

Figure S2. Pore size distribution of NU-1000 and Cyt c@NU-1000.

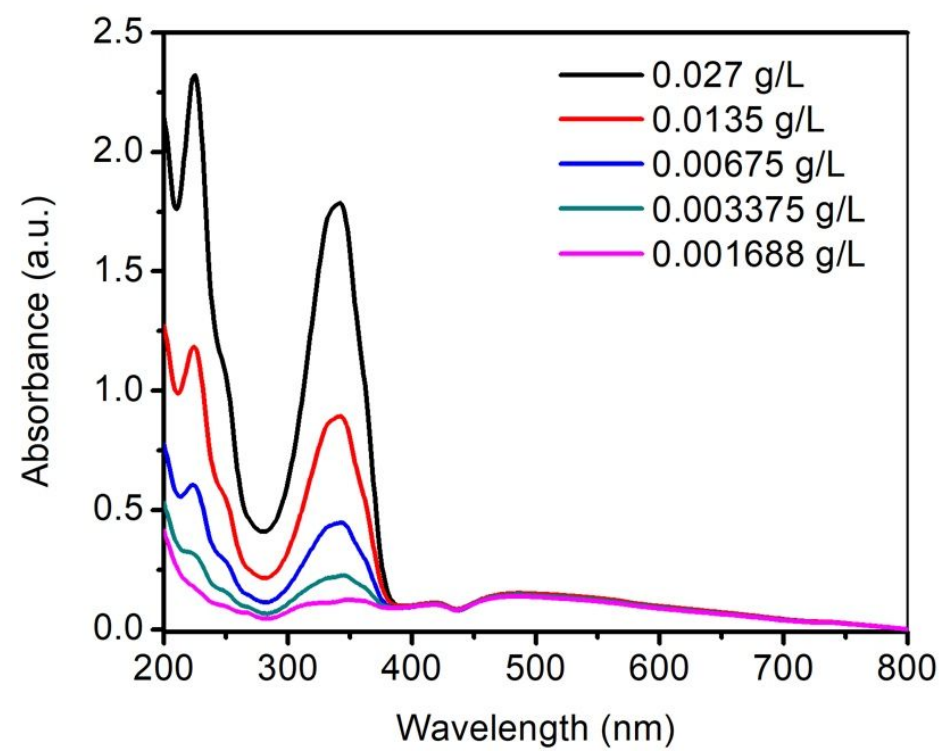

Figure S3. Absorption spectrum of ABTS at different concentrations. 


\section{Supporting Information}

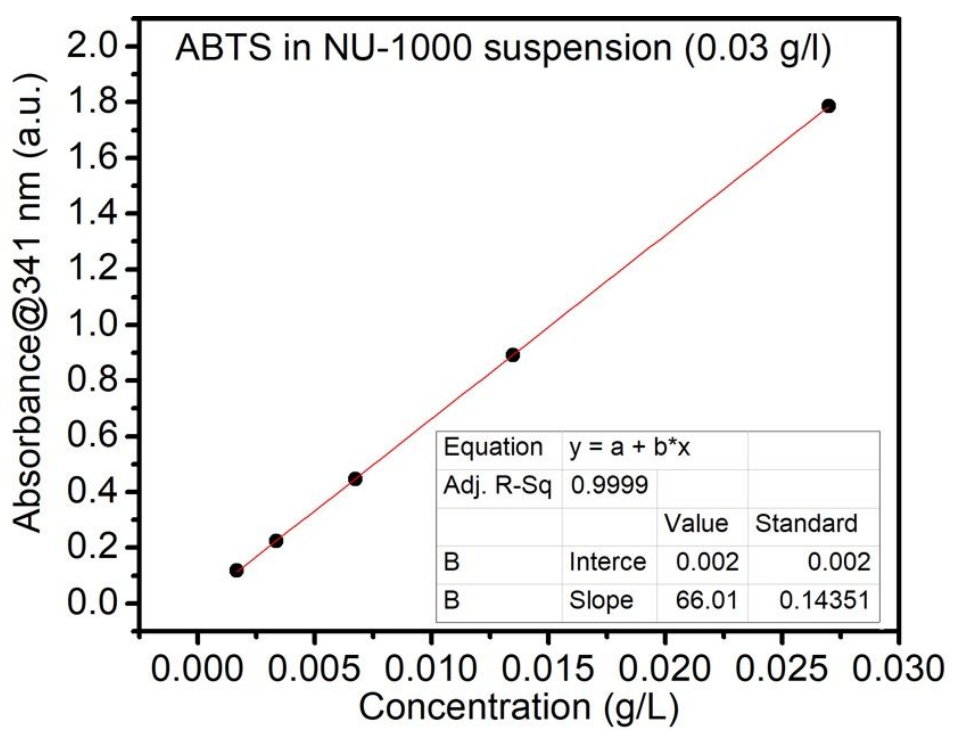

Figure S4. Standard curve of ABTS in $0.03 \mathrm{~g} / \mathrm{L}$ MOF suspension measured by UV-vis.

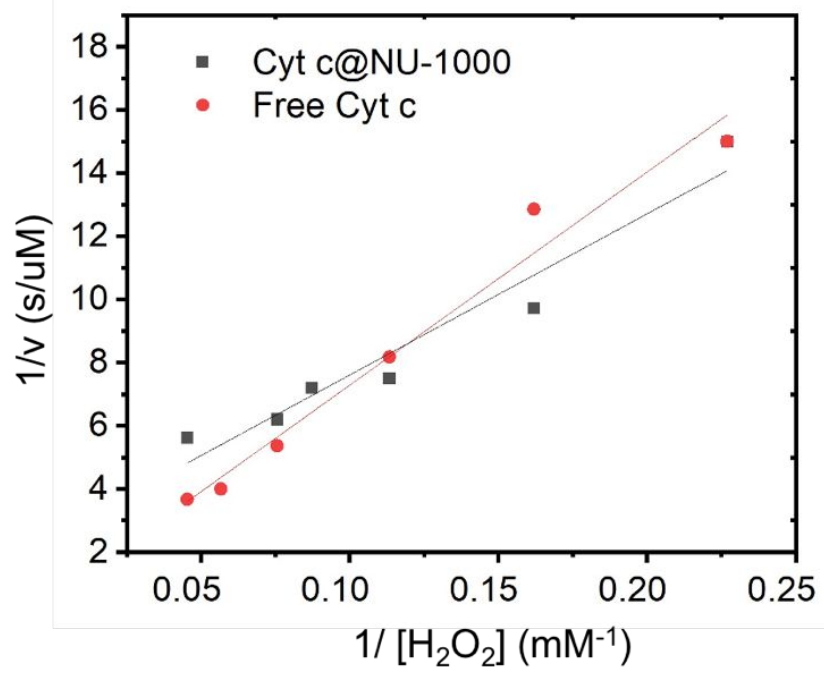

Figure S5. Double reciprocal plots of $\mathrm{H}_{2} \mathrm{O}_{2}$ concentrations with activities of Cyt c@NU1000 and free Cyt c. 


\section{Supporting Information}

(a)

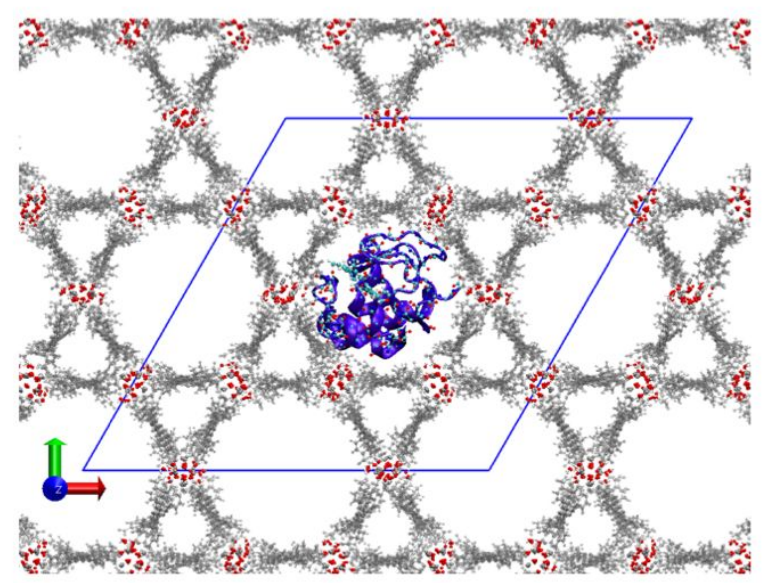

(b)

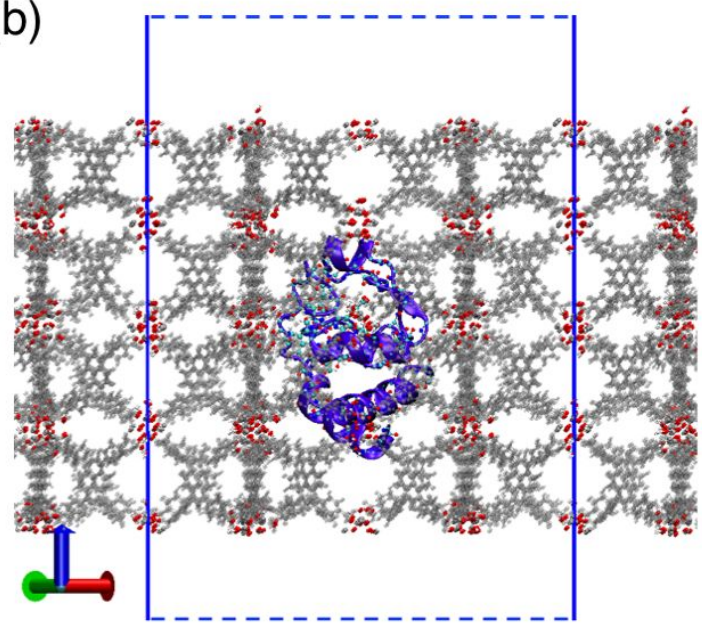

Figure S6. (a) Top and (b) side views of the simulation setup for Cyt c encapsulated inside MOF NU-1000. The MOF linkers are colored in grey whereas the protein backbone is in purple.

(a)

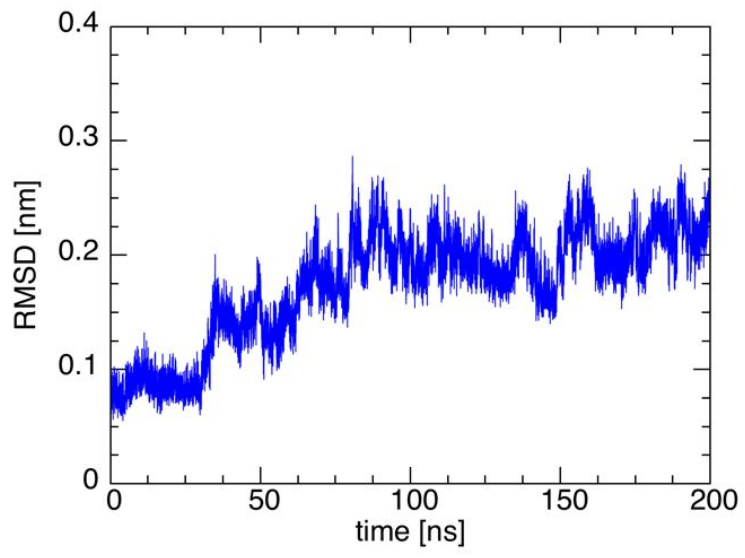

(b)

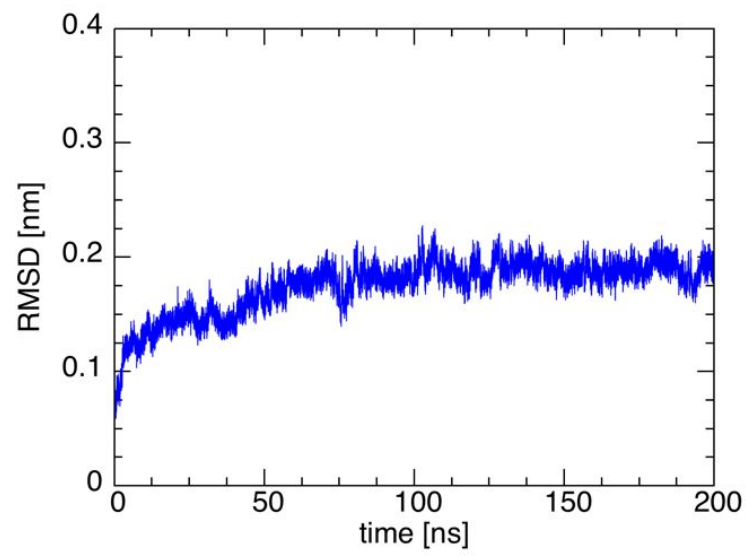

Figure S7. Root-mean-square deviation (RMSD) for the Cyt c backbone during the 200-ns production runs (a) free and (b) inside the NU-1000. 


\section{Supporting Information}

(a)

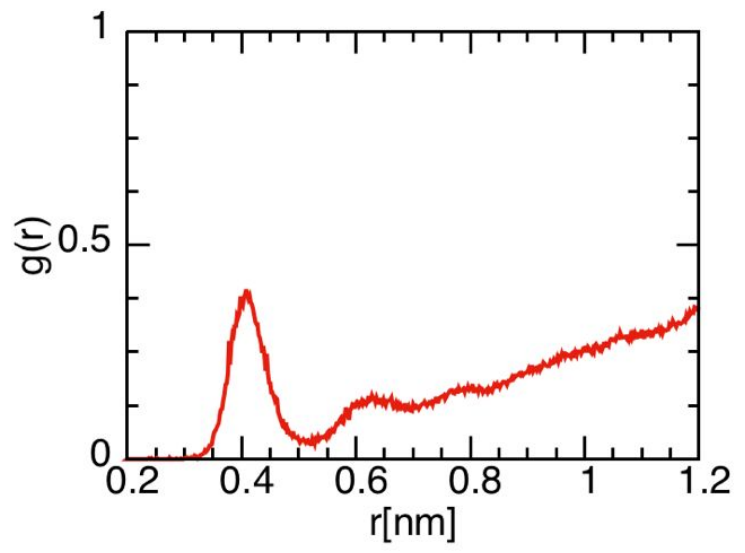

(b)

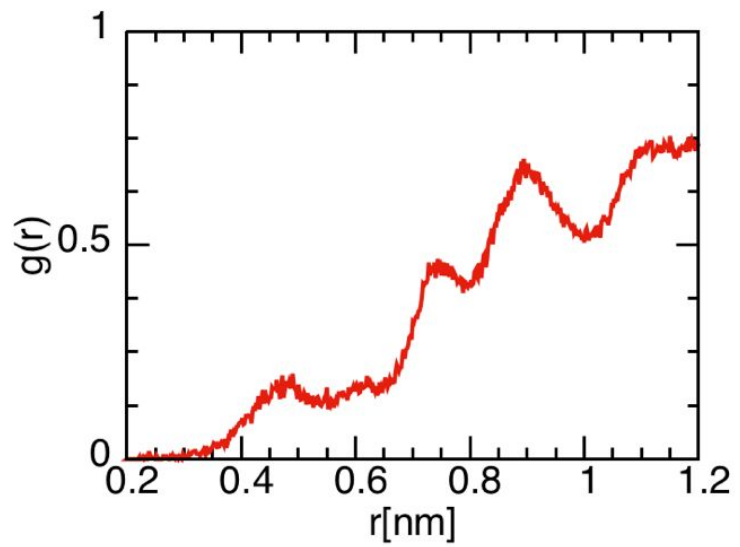

Figure S8. Radial distribution function $\mathrm{g}(\mathrm{r})$ of the water oxygen atoms as a function of the distance to heme Fe atom for Cyt c (a) free and (b) inside the NU-1000.

(a) Free Cyt C

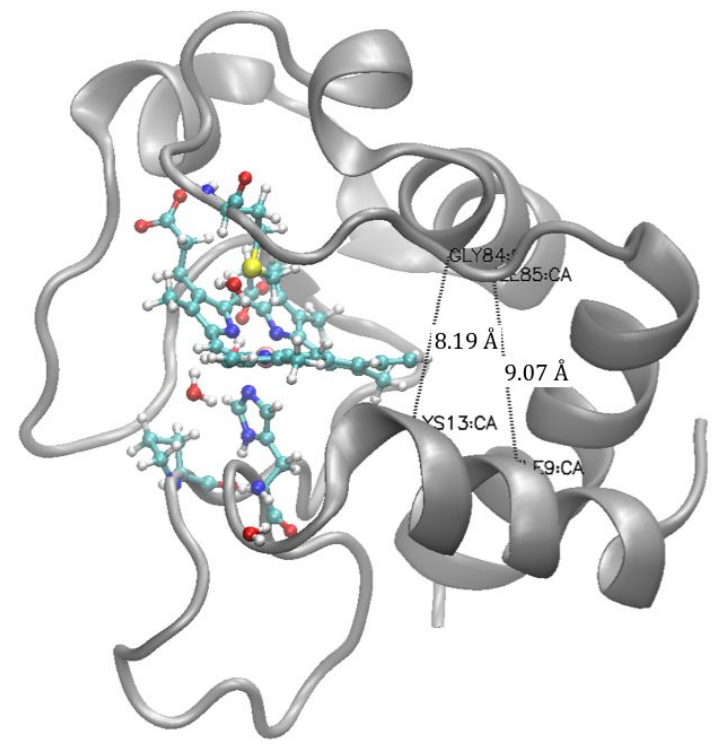

(b) Encapsulated Cyt c

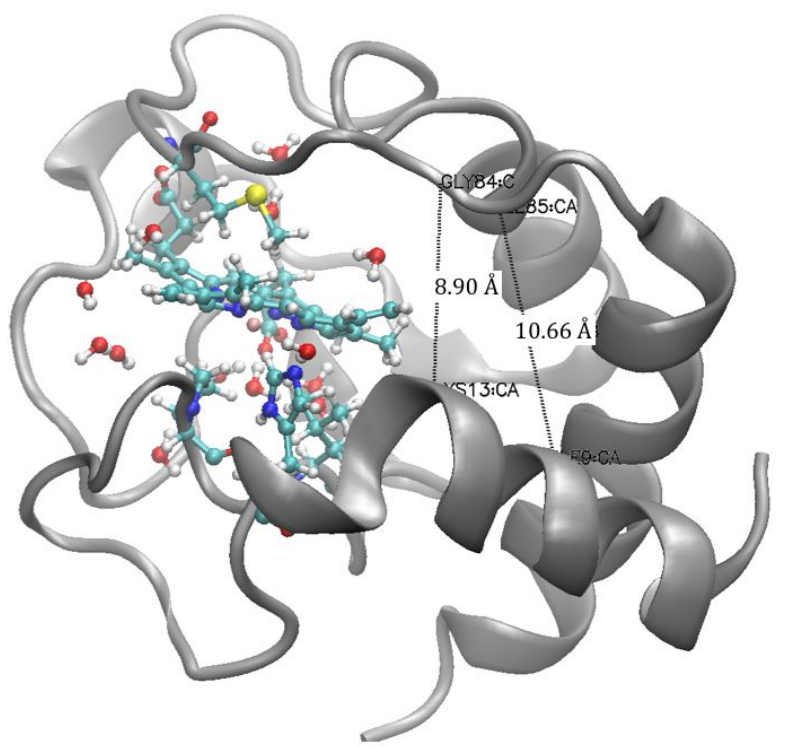

Figure S9. Instantaneous snapshots showing that the entrance to the Cyt $\mathrm{c}$ heme active site widens upon encapsulation by NU-1000. The separation distance between the backbone atoms Lys 13:C $\alpha$ to Gly84:C and between Ile85:C $\alpha$ to ILE9:CA of Cyt c (a) free and (b) inside the NU-1000. Confinement increases of the separation distances (partial unfolding of Cyt c) allowing a better access of water into the heme region. The water molecules are shown within $8 \AA$ of Fe. 


\section{Supporting Information}

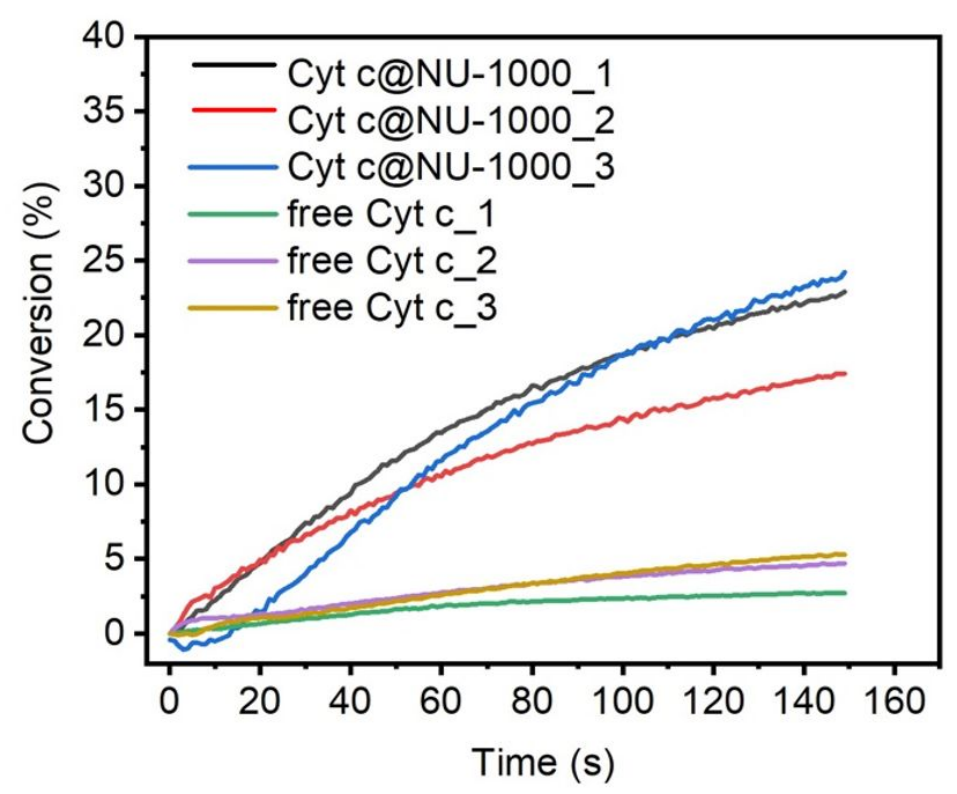

Figure S10. The oxidized ABTS in solution catalyzed by Cyt c and Cyt c@NU-1000.

\section{References}

1. Islamoglu, T.; Otake, K.-i.; Li, P.; Buru, C. T.; Peters, A. W.; Akpinar, I.; Garibay, S. J.; Farha, O. K., Revisiting the structural homogeneity of NU-1000, a Zr-based metal-organic framework. CrystEngComm 2018, 20 (39), 5913-5918.

2. Maximova, K.; Wojtczak, J.; Trylska, J., Enzyme kinetics in crowded solutions from isothermal titration calorimetry. Anal. Bioanal. Chem. 2019, 567, 96-105. 